\title{
Editorial
}

\section{Visual electrophysiology in clinical practice: current status and future prospects in Sri Lanka.}

P. Dahanayake $\mathrm{e}^{1,2^{*}}$

${ }^{1}$ Department of Physiology, Faculty of Medicine, University of Peradeniya, Sri Lanka.

${ }^{2}$ Teaching Hospital, Peradeniya, Sri Lanka.

\begin{abstract}
Visual electrophysiological evaluation includes a battery of tests that can be used to assess the function of the visual pathway from the photoreceptors of the retina to the visual cortex of the brain. Information obtained from these diagnostic tests helps to establish an accurate diagnosis or may rule out associated ophthalmic disorders. These tests could be used to diagnose a variety of inherited retinopathies, optic neuropathies, and visual defects caused by toxic substances, inflammatory conditions, ischaemic conditions and traumatic lesions. Though visual electrophysiology is not yet well established in Sri Lankan clinical practice, it is considered a valuable clinical companion predominantly in the fields of ophthalmology and neurology worldwide. These tests provide valuable evidence to correlate the functional abnormalities with the structural derangements of the visual system. Thus, this objective assessment of visual function is an essential component that has to be incorporated into our clinical practice in the near future to alleviate the increasing burden of visual loss.
\end{abstract}

Keywords: Visual electrophysiology, VEP, ERG, EOG, Visual disorders, Sri Lanka

Copyright: (C) 2021 Dahanayake P. (c) (i) This is an open-access article distributed under the Creative Commons Attribution License, which permits unrestricted use, distribution, and reproduction in any medium, provided the original work is properly cited.

Funding: None

Competing interest: None

Received: 01.12 .2021

Accepted revised version: 15.12 .2021

Published: 31.12 .2021

*ه Correspondence: padminid@pdn.ac.lk, iD https://orcid.org/0000-0001-8564-0637

Cite this article as: Dahanayake $P$, Visual electrophysiology in clinical practice: current status and future prospects in Sri Lanka. Anuradhapura Medical Journal 2021; 15 (2): 1-5, DOI:

http://doi.org/10.4038/amj.v15i2.7708

Visual electrophysiology is an advanced, noninvasive technique that is used in the clinical practice of ophthalmology and neurology. It is of utmost importance in the diagnosis of diseases affecting the visual pathway. This technique can be used to assess the functional integrity of the retina and the optic nerve pathway objectively. It bridges the structural information provided by imaging and clinical observations. Clinical visual electrophysiology, therefore, is considered an essential component of ophthalmic practice [1]. Visual electrophysiology consists of a range of investigations that record bioelectrical activity elicited by visual stimulation. Clinical standards of most of these tests are updated periodically by the international society of clinical electrophysiology of vision (ISCEV).

At present, it is estimated that nearly one billion people are visually impaired globally [2]. In Sri Lanka, the prevalence of visual impairment is estimated at $1.0 \%$ [3]. Most visual disorders are due to lesions in the optic nerve pathway and retina [4]. However, it has been identified that $90 \%$ of this visual impairment is avoidable if detected and treated as early as possible. Thus, a systematic approach using necessary investigations is essential to confirm the diagnosis. Though many 
sophisticated techniques are available to detect and evaluate structural integrity, only a very few techniques exist to assess the functional integrity of the visual pathway. To date, visual electrophysiology is the key test used to assess the functional integrity of the visual system. Clinical electrophysiological testing incorporates a range of tests and provides an objective indication of the function relating to different locations and cell types within the visual system. Visual evoked potentials (VEP) assess the functional integrity of the postretinal pathways, full-field electroretinography (ffERG) assesses the functional integrity of the retina, and electro-oculography (EOG) assesses the functional integrity of the interface between photoreceptors and retinal pigment epithelium (RPE). Pattern electroretinography (PERG) is a response of the central retina which is derived largely from the macular retinal ganglion cells and photoreceptors and is important in differentiating between maculopathy and generalized retinopathy [5]. Multifocal electroretinography (mfERG) allows local ERG responses to be recorded simultaneously from many regions of the retina. Therefore, it is evident that visual electrophysiology plays a distinct role in the ophthalmology arena and is increasingly used by ophthalmologists for accurate diagnosis worldwide.

\section{What is the current status of visual electrophysiology in Sri Lanka?}

Unfortunately, only a very few health care centres carry out these tests in Sri Lanka. However, the whole range of tests is still done only in one or two centres. Also, no consensus guidelines have been devised to specify how and when these techniques can be applied for the diagnostic work-up of patients with various visual disorders. However, recently, few visual electrophysiological studies have been carried out in our patients with different visual disorders that are the prevalent causes leading to significant visual impairment in Sri Lanka. In those studies, visual electrophysiological findings have been measured and interpreted in patients with optic neuritis, diabetic retinopathy, inherited retinopathies, toxic retinopathies, and many other visual defects. The findings emphasize the importance of introducing these tests to the current ophthalmology as well as neurology practice.
One such study revealed that the clinical profile of optic neuritis in Sri Lankans is different from the classic Western-type of optic neuritis, which is associated with multiple sclerosis. Interestingly, it was noted that our clinical profile is similar to the Asian-type optic neuritis with regard to the high occurrence of optic disc oedema in fundoscopy $(66.7 \%)$, preponderance of bilateral occurrence (41.1\%), and low occurrence of eye pain $(58.8 \%)$ at presentation. It is a well-known fact that VEP is a highly sensitive test for detecting demyelinating optic nerve damage. The involvement of the macula region of the retina in optic neuritis has not been extensively studied. Findings of that study have shown that the ganglion cells of the macula region could be affected in optic neuritis as indexed by abnormalities in the N95 component of PERG, which is compatible with the concept of retrograde degeneration of the ganglion cells occurring in optic neuritis [6].

As per the optic neuritis treatment trial (ONTT), treatment with combined intravenous and oral corticosteroids will accelerate the short-term visual recovery in optic neuritis $[7,8]$. Since then, that has become the standard treatment. Given that VEPs were not used as an outcome measure in this ONTT study, or even in other studies done in this regard to assess the efficacy of combined corticosteroid treatment, pattern reversal VEPs (PRVEP) have been used to assess the short-term recovery of visual conduction pathways in a cohort of Sri Lankan patients with optic neuritis who underwent treatment with combined ONTT regimen [9-12]. In this follow up study, interestingly, it was observed that there is a marked trend of improvement in P100 latency values in PRVEP in affected eyes in the ONTT combined regimen treated group immediately one month after the treatment, and further improvement was observed in 3 months after the pre-treatment, baseline assessment [13]. Therefore, it can be assumed that ONTT combined regimen could have accelerated the rate of recovery faster as shown by the original ONTT. This would be a significant observation that can be considered when deciding the management strategy of the patients clinically.

Diabetic retinopathy is a devastating complication of diabetes mellitus. In Sri Lanka, nearly one-third of diabetic patients have retinopathy which amounts to a significant burden to the health care sector. In 
another visual electrophysiological study done in a group of Sri Lankan diabetic patients, it was shown that one in three patients who do not show fundoscopic abnormalities have functional abnormalities of the retina. In other words, it is estimated that one-third to three-fourths of patients with newly diagnosed diabetes mellitus show electrophysiological evidence of RPE dysfunction even before developing clinically evident retinopathy or visual impairment [14]. These findings indicate that visual electrophysiological tests can be utilized as a sensitive tool to detect early diabetic retinopathy before developing fundoscopic changes.

As genetic diagnosis is not readily available in Sri Lanka at present to detect inherited retinopathies, a combination of clinical features, retinal imaging, and the findings of the visual electrophysiological tests would be of immense value to confirm the diagnosis. These tests would aid early diagnosis and clinical management of these patients, and also, they could be used as a screening tool of family members of patients who are suspected of having inherited retinal disorders. Also, the progression of the disease through different layers of the retina could be assessed using these visual electrophysiological tests in a detailed manner $[15,16]$. Best vitelliform macular dystrophy, adult vitelliform macular dystrophy, retinitis pigmentosa, and Stargardt's disease are some of the common inherited retinopathies in Sri Lanka.

Though it is not revealed extensively, exposure to some toxic substances such as organophosphorus compounds (OP) could cause significant retinal damage. In these instances, objective assessment of visual function is significant, predominantly where structural changes in the visual system have not yet occurred, as there may be a window of opportunity to mitigate or reverse visual impairment [17]. Since OP poisoning is a leading cause of mortality and morbidity in Sri Lanka, it is important to investigate the ophthalmic derangements caused by such substances as well [18]. OPs inhibit acetylcholinesterase (AChE) by phosphorylating that enzyme. There are nicotinic ACh receptors on RPE cells, and, hence, ACh is likely to be present in the outer photoreceptor segment [19]. This makes the RPE a potential target in OP poisoning, but only a few human studies have examined RPE deficits in OP poisoning. A toxicological study conducted in a tertiary care centre in the Central province recently found out that acute OP poisoning seems to affect the functions of the RPE, and those changes seem to outlast the cholinergic phase of poisoning [20]. This early detection of visual abnormalities is important to decide on the subsequent management of OP poisoned patients, which is still a neglected part of their management protocol. In addition, it is noteworthy to assess the visual electrophysiological changes caused by other toxic substances as well as drugs as an extension to this study.

\section{What lies ahead? Future prospects of visual electrophysiology in Sri Lankan context}

Based on the current status, it is clearly evident that visual electrophysiological evaluation is an essential diagnostic method that has to be integrated into our clinical practice. Also, these tests need to be more widely available in different health care centres around the country. When this is established, innovations of clinical measures such as wireless electrodes and advanced signal processing can be introduced gradually. Also, it has to be mentioned that visual electrophysiology has an important role in pediatric ophthalmology as it provides a noninvasive modality for examination. Early diagnosis of these conditions will aid in the early visual rehabilitation of the child. Considering all these facts, as many international studies highlight, clinical visual electrophysiology has an exciting and evolving role in future ophthalmological and neurological care in Sri Lanka too.

\section{References}

1. Hamilton R. Clinical electrophysiology of vision - commentary on the current status and future prospects. Eye. 2021;35(9):2341-3. doi: https://doi.org/10.1038/s41433-021-01592-0

2. Pascolini D, Mariotti SP. Global estimates of visual impairment: Br J Ophthalmol. 2012;96(5):6148 doi: 10.1136/bjophthalmol-2011-300539 
3. Gilbert C, Edusuriya K, Murthy GVS, Pant HB, Schmidt E, Senanayake S, et al. Prevalence, causes and magnitude of functional low vision in Sri Lanka: results from a national population based survey. Ceylon Med J. 2018;63(5):s40-s4. doi: https://doi.org/10.4038/cmj.v63i5.8741

4. WHO. Blindness and vision impairment: World Health Organization; 2020 [cited 10 ${ }^{\text {th }}$ January 2021]. Available from: https://www.who.int/news-room/fact-sheets/detail/blindness-and-visualimpairment.

5. Holder GE. Pattern electroretinography (PERG) and an integrated approach to visual pathway diagnosis. Prog Retin Eye Res. 2001;20(4):531-61. doi: 10.1016/s1350-9462(00)00030-6

6. Dahanayake D, Pathirage L, Hangilipola A, Baminiwattha D, Senanayake S, weerasinghe VS. Combined use of pattern electroretinography (PERG) and pattern reversal visual evoked potentials (PVEP) in optic neuropathies. Journal of the college of ophthalmologists of Sri Lanka. 2017;23(2):55-8.

7. Beck RW, Trobe JD. What We Have Learned from the Optic Neuritis Treatment Trial. Ophthalmology. 1995;102(10):1504-8. doi: 10.1016/s0161-6420(95)30839-1

8. Beck RW, Cleary PA, Anderson MM, Keltner JL, Shults WT, Kaufman DI, et al. A randomized, controlled trial of corticosteroids in the treatment of acute optic neuritis. NEJM. 1992;326(9):581-8. doi: 10.1056/NEJM199202273260901

9. Trauzettel-Klosinski S, Diener HC, Dietz K, Zrenner E. The effect of oral prednisolone on visual evoked potential latencies in acute optic neuritis monitored in a prospective, randomized, controlled study. Doc Ophthalmol. 1995;91(2):165-79. doi: 10.1007/BF01203696

10. Kapoor R, Miller DH, Jones SJ, Plant GT, Brusa A, Gass A, et al. Effects of intravenous methylprednisolone on outcome in MRI-based prognostic subgroups in acute optic neuritis. Neurology. 1998;50(1):230.doi: 10.1212/wnl.50.1.230

11. Morrow SA, Fraser JA, Day C, Bowman D, Rosehart H, Kremenchutzky M, et al. Effect of treating acute optic neuritis with bioequivalent oral vs intravenous corticosteroids: a randomized clinical trial. JAMA Neurol. 2018;75(6):690-6. doi: 10.1001/jamaneurol.2018.0024

12. Sethi H, Menon V, Sharma P, Khokhar S, Tandon R. Visual outcome after intravenous dexamethasone therapy for idiopathic optic neuritis in an Indian population: A clinical case series. Indian J Ophthalmol. 2006;54(3):177-83. doi: 10.4103/0301-4738.27069

13. Dahanayake P, Dassanayake TL, Pathirage M, Senanayake S, Sedgwick M, Weerasinghe VS. The effect of optic neuritis treatment trial (ONTT) combined corticosteroid regimen on pattern reversal visual evoked potentials: a prospective follow-up study. BMC Ophthalmol. 2021;21(1):324. doi: 10.1186/s12886-021-02086-z

14. Dahanayake P, Dassanayake TL, Pathirage M, Senanayake S, Sedgwick M, Weerasinghe V. Detection of early diabetic retinopathy using visual electrophysiological tests. Int J Diabetes Dev Ctries. 2021. doi: 10.1007/s13410-020-00916-2

15. Dahanayake DWP., Pathirage LPMMK., Shiroma RGL., Senanayake S., VS W, editors. Combined use of pattern electroretinography (PERG) and electro-oculography (EOG) in retinal pigment epithelial disorders: preliminary data. Peradeniya university international research sessions (iPurse); 2017 .

16. Dahanayake DWP., Pathirage LPMMK., Shiroma RGL., Senanayake S., Weerasinghe VS. A study of electro-oculography (EOG) in patients with retinal disorders. 39th Annual academic sessions of the Kandy Society of Medicine; 2017.

17. O'Neill EK, Smith R. Visual electrophysiology in the assessment of toxicity and deficiency states affecting the visual system. Eye. 2021;35(9):2344-53. doi: 10.1038/s41433-021-01663-2

18. Gunnell D, Eddleston M, Phillips MR, Konradsen F. The global distribution of fatal pesticide selfpoisoning: Systematic review. BMC Public Health. 2007;7(1):357. doi: 10.1186/1471-2458-7-357 
19. Maneu V, Gerona G, FernÁNdez L, Cuenca N, Lax P. Evidence of alpha 7 nicotinic acetylcholine receptor expression in retinal pigment epithelial cells. Vis Neurosci. 2010;27(5-6):139-47.doi : 10.1017/S0952523810000246

20. Dahanayake P, Dassanayake TL, Pathirage M, Colombage A, Gawarammana IB, Senanayake S, et al. Dysfunction in macula, retinal pigment epithelium and post retinal pathway in acute organophosphorus poisoning. Clin Toxicol. 2020:1-7. doi: 10.1080/15563650.2020.1771359 